\title{
A particle swarm based hybrid system for imbalanced
} medical data sampling

\author{
Pengyi Yang*1,2, Liang Xu' ${ }^{3}$, Bing B Zhou*1, Zili Zhang ${ }^{3,4}$ \\ and Albert Y Zomaya ${ }^{1,5}$
}

Addresses: ${ }^{1}$ School of Information Technologies (J12), The University of Sydney, NSW 2006, Australia, ${ }^{2}$ National ICT Australia, Australian Technology Park, Eveleigh, NSW 2015, Australia, ${ }^{3}$ Faculty of Computer and Information Science, Southwest University, CQ 400715, PR China, ${ }^{4}$ School of Information Technology, Deakin University, Geelong, VIC 3217, Australia and ${ }^{5}$ Sydney Bioinformatics Center and the Center for Mathematical Biology, The University of Sydney, NSW 2006, Australia

E-mail: Pengyi Yang* - yangpy@it.usyd.edu.au; LiangXu - sumkire@swu.edu.cn; Bing B Zhou* - bbz@it.usyd.edu.au; Zili Zhang - zili.zhang@deakin.edu.au; Albert Y Zomaya - zomaya@it.usyd.edu.au

${ }^{*}$ Corresponding author

from Asia Pacific Bioinformatics Network (APBioNet) Eighth International Conference on Bioinformatics (InCoB2009)

Singapore 7-II September 2009

Published: 3 December 2009

BMC Genomics 2009, I0(Suppl 3):S34 doi: 10.1186/147|-2164-10-S3-S34

This article is available from: http://www.biomedcentral.com/I47I-2/64/I0/S3/S34

(C) 2009 Yang et al; licensee BioMed Central Ltd.

This is an open access article distributed under the terms of the Creative Commons Attribution License (http://creativecommons.org/licenses/by/2.0), which permits unrestricted use, distribution, and reproduction in any medium, provided the original work is properly cited.

\begin{abstract}
Background: Medical and biological data are commonly with small sample size, missing values, and most importantly, imbalanced class distribution. In this study we propose a particle swarm based hybrid system for remedying the class imbalance problem in medical and biological data mining. This hybrid system combines the particle swarm optimization (PSO) algorithm with multiple classifiers and evaluation metrics for evaluation fusion. Samples from the majority class are ranked using multiple objectives according to their merit in compensating the class imbalance, and then combined with the minority class to form a balanced dataset.

Results: One important finding of this study is that different classifiers and metrics often provide different evaluation results. Nevertheless, the proposed hybrid system demonstrates consistent improvements over several alternative methods with three different metrics. The sampling results also demonstrate good generalization on different types of classification algorithms, indicating the advantage of information fusion applied in the hybrid system.

Conclusion: The experimental results demonstrate that unlike many currently available methods which often perform unevenly with different datasets the proposed hybrid system has a better generalization property which alleviates the method-data dependency problem. From the biological perspective, the system provides indication for further investigation of the highly ranked samples, which may result in the discovery of new conditions or disease subtypes.
\end{abstract}




\section{Background}

One of the difficulties in medical and biological data analysis is the highly skewed class distribution of different sample types. This could happen when special cases or "positive" samples are of limited size, while control or "negative" samples are more abundant [1-4]. Sometimes, disease samples are divided into subtypes, with some of which are common while others are very rare. Samples from those rare subtypes are represented as minority classes which also cause the imbalance of the class distribution [5]. Here the challenge is how to precisely and correctly classify the minority samples (rare cases) because they often carry important biological implications but tend to be ignored by the classification model which is overwhelmed by the majority samples. In data mining community this problem is known as imbalanced data classification [6] and recently received an increasing attention for its practical importance.

There are mainly two strategies in dealing with imbalanced data learning: via sampling and via cost-sensitive learning. Although cost-sensitive learning does not modify the data distribution or introduce duplicated samples, it requires the right cost-metric to assign different penalties for misclassification of different sample types. However, the correct cost-metric is often unknown a priori for a given dataset, and an improper cost-metric can significantly degenerate the classification accuracy [7]. Recently, much effort has been made for developing new sampling strategies [8-10].

Data sampling strategies can often be categorized into two groups: oversampling and undersampling. In oversampling, the samples in the minority class are increased to match the samples of the majority class, while in undersampling the samples in the majority class are decreased to match the samples of the minority class. The classical or "naive" method is to randomly select samples from minority class and use the selected samples to increase the size of the minority class for oversampling (random oversampling) or to randomly select samples from majority class and remove them so as to decrease the sample size for undersampling (random undersampling) [11]. More advanced methods attempt to employ certain intelligent strategies such as clustering [10], working on the decision boundary [12] or synthesizing new examples based on the data characteristics $[8,13]$. There are also many distance-based methods which try to select the samples with the nearest distance or farthest distance between the majority class and the minority class [14]. However, currently there is no clear way to determine which rule should be followed, and simply applying random sampling often beats those "smart" methods [15]. The unsuccessful experiences imply that those methods are largely data-depended. Therefore, designing more flexible and better generalized algorithms which are self-adaptable to different data patterns in imbalanced data sampling and accurate model construction is clearly a desirable goal. This is particularly true in medical data classification and diagnosis because a false positive prediction will cause unwarranted worries while a false negative prediction will increase the risk of missing medical attention.

In previous work, Zhang and Yang successfully applied a genetic ensemble hybrid system to the feature selection of high-dimensional data [16]. If we convert the question by treating samples as features and re-adopt such kind of feature selection methods to select a subset of samples in majority class for building a balanced classification model, will such formulation lead to a better balanced classification result? This study is set out to investigate this quest. Here we formulate the problem as an optimization process and employ the particle swarm optimization (PSO) algorithm as the sample selection strategy $[17,18]$. Multiple classification algorithms with several most indicative metrics for imbalance classification measurement are used as multiple objectives to guide the sample selection process. Although there are continuing debates on which technique is better [19], undersampling is often preferred because no duplicated samples are introduced [20,21]. Therefore, our study will concentrate on selecting an optimal subset of majority samples and combine them with the minority samples for building a balanced classification model. Nevertheless, the proposed algorithm can be easily applied to oversampling by changing the target as minority samples.

\section{Methods}

\section{System overview}

The problem of using highly imbalanced dataset for pattern recognition is that the classification model built on the training data tends to be biased on preferring the majority class while ignoring the samples from the minority class. Data sampling method tries to remedy the skewed class distribution by either increasing the sample size of minority class or decreasing the sample size of majority class. However, algorithms that modify the sample distribution with greedy measures can introduce undesired bias. In this study we re-apply the techniques in feature selection to data sampling using a PSO based hybrid system. The schematic flow of sampling and evaluation processes in our hybrid system is illustrated in Figure 1.

As can be seen, the work flow can be divided into two steps, namely, sampling and evaluation. For a given dataset, an external 3-fold stratified cross validation is applied to partition the dataset into external training sets (sampling sets) and external test sets (evaluation sets). 


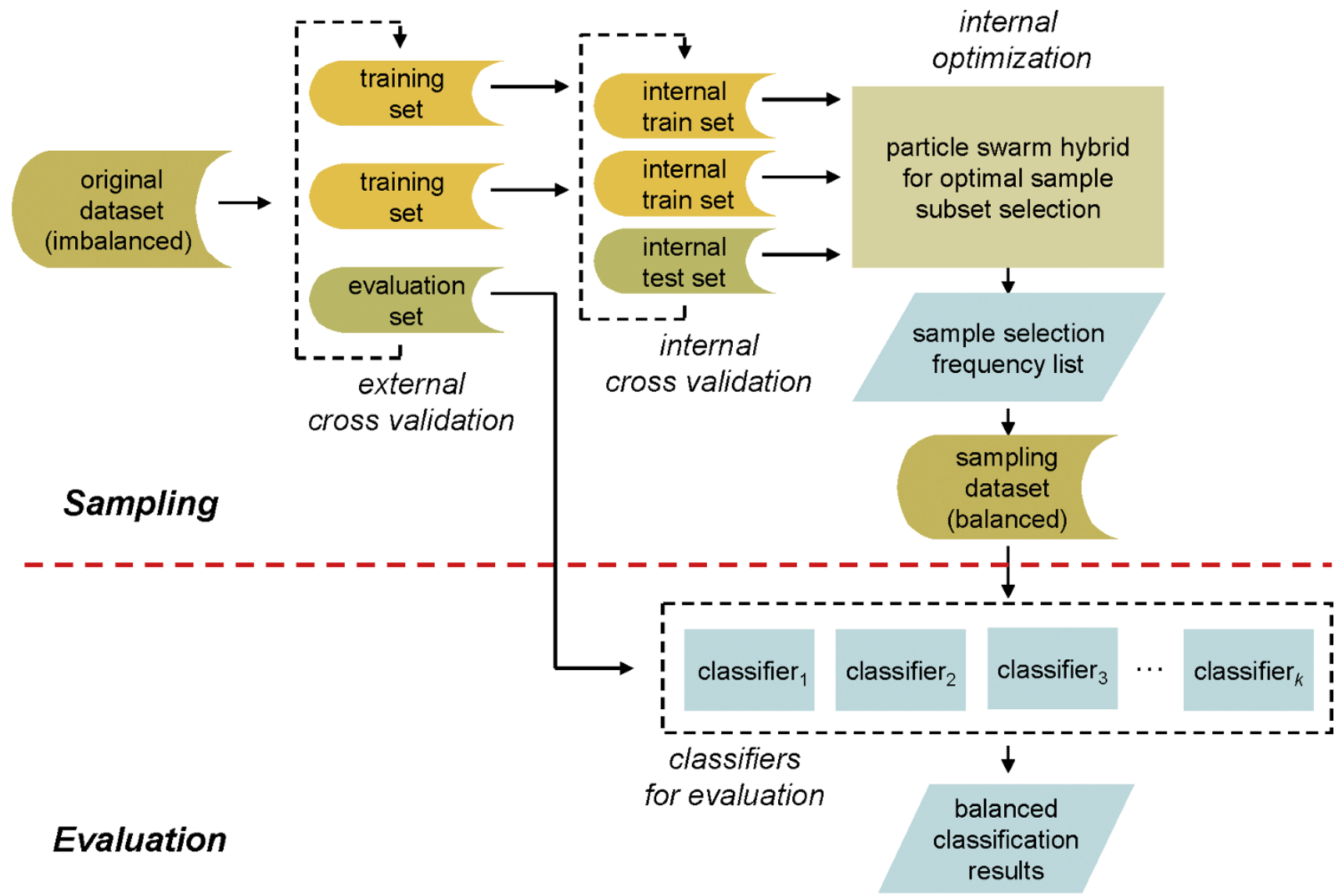

Figure 1

Figure I

Schematic flow chart of sampling and evaluation processes. The original imbalanced dataset are split to training and test sets with an external stratified cross validation. The sampling process is then conducted on an internal stratified cross validation for creating a balanced training set. The classification models are built on the balanced training set and the test set from the external cross validation is classified using the obtained classification models.

Then, the external training sets are further partitioned with an internal 3-fold stratified cross validation, which gives the internal training sets and internal test sets. The internal training sets are used for sampling, while the internal test sets are used for guiding the optimization process. The external test sets are reserved for evaluation of the balanced dataset and is excluded from the sampling procedure.

In the sampling procedure, the PSO hybrid system is used to evaluate the merit of each sample from the majority class in compensating the class imbalance. This is accomplished by generating different sample subsets of majority class and combining them with samples from the minority class for classification model construction and then for internal test fold classification. Those subsets that can create more accurate classification models are favored and optimized in each PSO iteration. When the termination criterion is met, selected samples from the last iteration are ranked by their selection frequency. After the sample selection frequency list is obtained, a balanced dataset can be created by combining the highly ranked samples of majority class with samples of minority class. In the evaluation step, different classification models are created using the balanced dataset generated by PSO hybrid system, and the external evaluation dataset is applied to evaluate the classification accuracy with different evaluation metrics. Such a training and evaluation process keeps the evaluation dataset for independent validation, which provides an unbiased evaluation.

\section{Particle swarm based optimization}

Particle swarm optimization (PSO) is a new group of population-based algorithms which uses the idea of social communication and historical behaviors to adjust the optimization process [17]. It possesses the advantages such as high-performance and global optimization, which make it very popular in many biological related 
applications. Specifically, Lee combined PSO with Genetic Algorithm (GA) and Support Vector Machine (SVM) for gene selection of microarray data [22], Xu et al. used PSO to optimize the structure of Recurrent Neural Network (RNN) in gene network modeling [23], while Rasmussen and Krink applied PSO for Hidden Markov Model optimization in multiple sequence alignment [24]. In our system, a binary version of PSO (BPSO) $[18,25]$ is employed for a new application, in which BPSO is hybridized with multiple classifiers and metrics for data sample selection and ranking.

Figure 2 gives a graphical representation of this particle swarm based hybrid module. In this module, different sample subsets are encoded as particles, and each particle is evaluated by multiple classifiers each with three evaluation metrics. The system seeks for the sample subsets that present good classification accuracy with not only a certain type of classifier but a wide range of them each provides the feedback using several evaluation criteria. The use of this hybrid system is justified with the argument that multiple criteria formulation is preferable than a single classification algorithm or evaluation metric because the results produced in this way will have a better generalization property.
Each sample of majority class in the training dataset is assigned an index in the particle space. The locus equals " 1 " if the sample is selected for building classification model or equals " 0 " if the sample is excluded from building the classification model. Suppose we have a population of $n$ particles, with $i$ be the index of a particle in the swarm $(i=1, \ldots, n), j$ be the index of dimension in the particle $(j=1, \ldots, m)$, and $t$ be the counter of iterations. The velocity of the $i$ th particle $v_{i, j}(t)$ and the position of this particle $x_{i, j}(t)$ is updated by BPSO with following equations:

$$
\begin{gathered}
v_{i, j}(t+1)=w \cdot v_{i, j}(t)+c_{1} R_{1} \cdot\left(\text { pbest }_{i, j}-x_{i, j}(t)\right)+c_{2} R_{2} \cdot\left(\text { gbest }_{i, j}-x_{i, j}(t)\right) \\
x_{i, j}(t+1)= \begin{cases}0: & \text { if random }() \geqslant S\left(v_{i, j}(t+1)\right) \\
1: & \text { if random }()<S\left(v_{i, j}(t+1)\right)\end{cases} \\
S\left(v_{i, j}(t+1)\right)=\frac{1}{1+e^{-v_{i, j}(t+1)}}
\end{gathered}
$$

where pbest $t_{i, j}$ and gbest $_{i, j}$ are the previous best position and the best position found by informants, respectively. random () is the pseudo-random number generator that creates uniform distribution between [0-1].

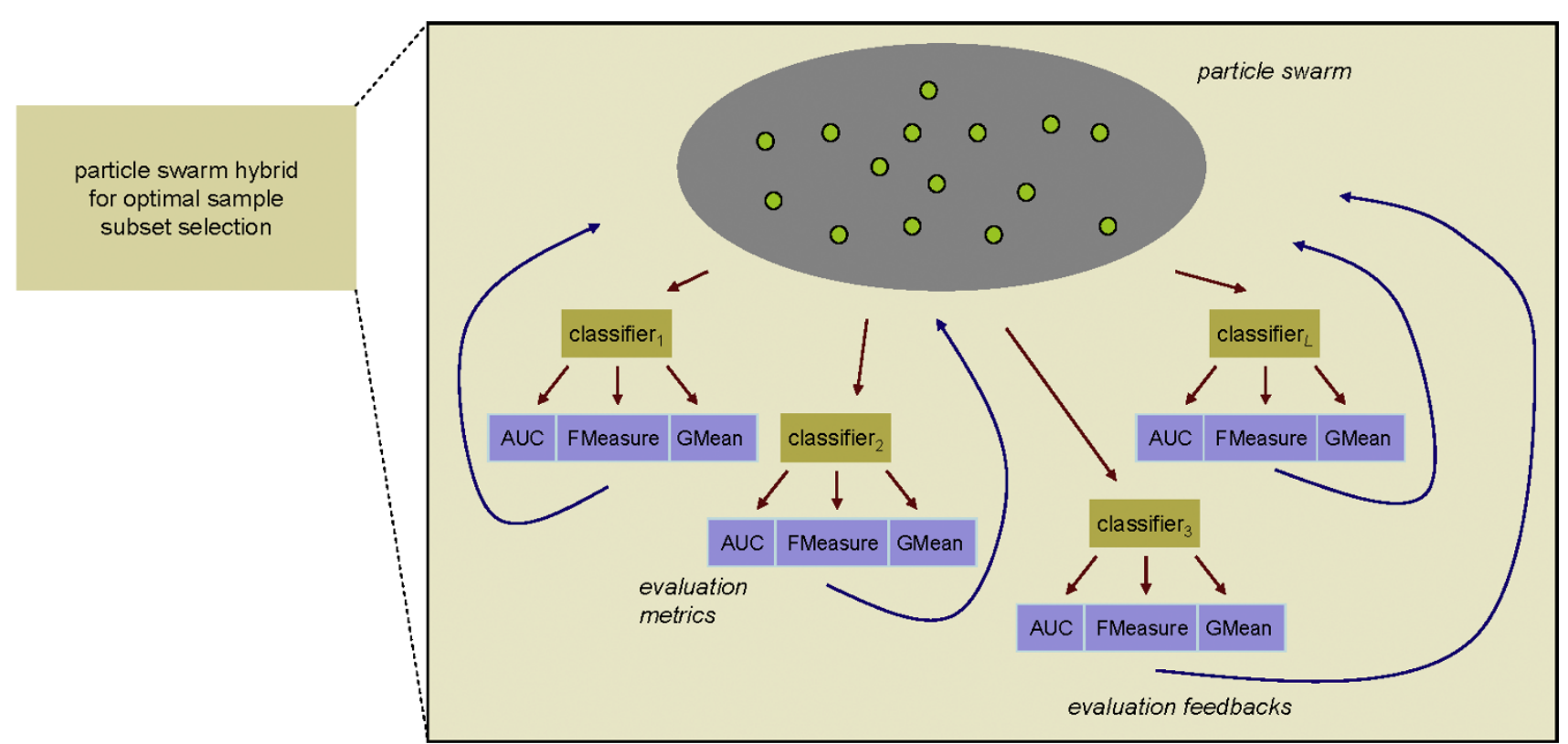

\section{Figure 2}

Particle swarm based hybrid module for data sampling. Multiple classification algorithms are used to guide the sampling process. Within each classification algorithm, three evaluation metrics are employed to evaluated the goodness of the sample subsets. PSO algorithm is used to optimize the sample subsets according to the evaluation results of each classification component. 


\section{Fitness and evaluation metrics}

Fitness function is the optimization guide of the BPSO. It governs the update of pbest $t_{i, j}$ and gbest $_{i, j}$. It has been pointed out that in the imbalanced data evaluation a simple classification accuracy is not an indicative measure because the accuracy value is profoundly influenced by the large class [13].

Alternatively, metrics including Area Under the ROC Curve (AUC), F-Measure (FMeasure), and Geometric Mean (GMean) are often chosen as more appropriate measures $[10,12,26,27]$. Here we combine multiple evaluation metrics in BPSO fitness function, which is defined as follows:

$$
\operatorname{overallFitness}(s)=\sum_{i=1}^{L} \frac{\text { futness }_{i}(s)}{L}
$$

where $L$ is the number of classifiers integrated in the hybrid system and fitness $(s)$ is formulated as follows:

$$
\text { fitness }_{i}(s)=w_{1} \cdot \operatorname{AUC}(s)+w_{2} \cdot F \text { Measure }(s)+w_{3} \cdot \operatorname{GMean}(s), \quad\left(\sum_{j=1}^{3} w_{j}=1\right)
$$

where $s$ is the sample subset to be evaluated. This fitness function is essentially a weighted combination of the above three evaluation metrics, $A U C(s)$ is calculated using Mann Whitney statistic [28], while FMeasure (s) and GMean(s) are calculated as follows:

$$
\begin{gathered}
\text { FMeasure }(s)=\frac{2 \times \text { Precision } \times \text { Recall }}{\text { Precision }+ \text { Recall }} \\
\text { GMean }(s)=\sqrt{\text { Sensitivity } \times \text { Specificity }}
\end{gathered}
$$

where each component in FMeasure(s) and GMean(s) is further defined as follows:

Precision:

$$
\text { Precision }=\frac{N_{T P}}{N_{T P}+N_{F P}}
$$

Sensitivity or Recall:

$$
\text { Sensitivity }=\text { Recall }=\frac{N_{T P}}{N_{T P}+N_{F N}}
$$

Specificity:

$$
\text { Specificity }=\frac{N_{T N}}{N_{T N}+N_{F P}}
$$

where $N_{T P}$ is the number of true positive, $N_{T N}$ is the number of true negative, $N_{F N}$ is the number of false negative, and $N_{F P}$ is the number of false positive.

\section{Classifiers}

One limitation of previous efforts on imbalanced data analysis is that most studies only focused on Decision Tree as evaluation criterion [6]. Instead of choosing certain type of classification algorithm for evaluation, multiple classifiers have been incorporated in our particle swarm based hybrid system. The reason of utilizing multiple classifiers is to balance multiple classification hypotheses so as to reveal true improvement of the sampling dataset.

Specifically, the classification algorithms employed in the hybrid system composition includes Decision Tree (J48), $k$-Nearest Neighbor $(k N N)$, Naive Bayes (NB), Random Forest (RF) and Logistic Regression (LOG). J48 is a widely used decision tree classifier. It approximates discrete-valued functions and a group of favorite features selected by the algorithm are used as the test points at the tree nodes. Each path of the node is then created for partitioning the value of the feature. $k \mathrm{NN}$ classifier calculates the similarity, which is called distance, of a given instance with the others and assign the given instance into the majority class which the $k$ most similar instances belong to. Such similarity can be defined as Euclidean distance, Manhattan distance or Pearson correlation. Naive bayes classifier bases its learning strategy on probability theory. It tries to estimate the distribution of the data and classify a sample by assigning the sample into a class with the highest probability. Random forest, as its name indicates, is a collection of decision trees [29]. Instead of using a single tree to make the classification, Random forest algorithm combines the decisions of several trees each trained on a feature subset of the original dataset. Lastly, the Logistic Regression classifier uses a logistic function to compute the coefficients of input features with respect to the class label. It has been used extensively in modeling binomially distributed data.

\section{Main loop}

Putting above components together, the BPSO based hybrid system can be summarized by pseudo-code in Figure 3.

\section{Experimental settings Datasets}

Four typical medical datasets are obtained from UCI Machine Learning Repository [30] and a genome wide association study (GWAS) dataset is obtained from the 


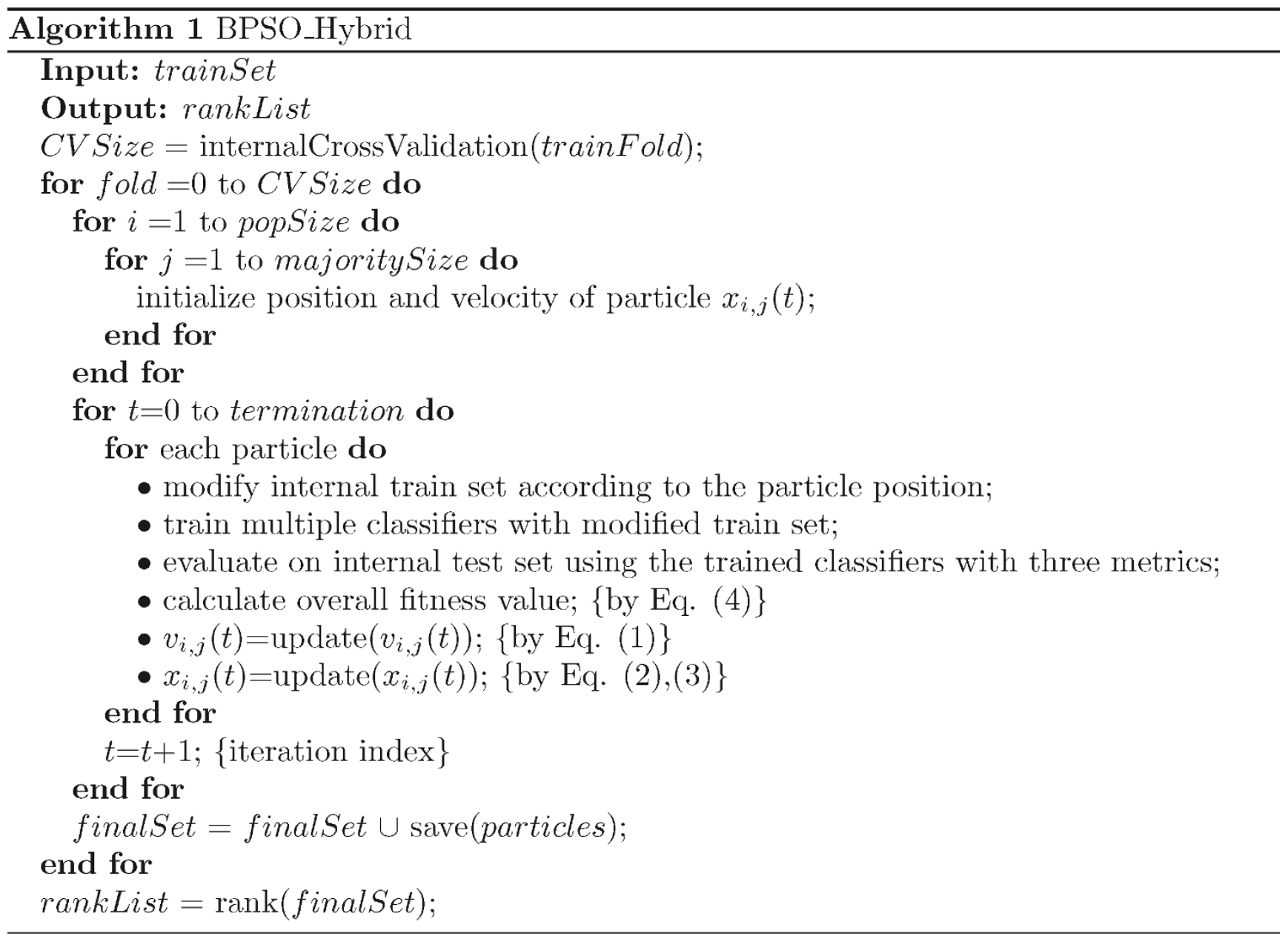

Figure 3

Figure 3

The main loop of the BPSO based hybrid algorithm.

genotyping of Single Nucleotide Polymorphisms (SNPs) of Age-related Macular Degeneration (AMD) [31].

For the medical data, the first dataset named "Blood" was generated by Blood Transfusion Service Center in Taiwan. It has 568 samples denoted as not donating blood and 180 as donating blood in March 2007, and the prevalence of the dataset is $24.1 \%$. The task is to classify these samples based on the information of blood donation frequency, recency etc. The second dataset, "Survival", was generated from the survey conducted on the survival of patients who had undergone surgery for breast cancer. It contains 225 patients who survived 5 years or longer and 81 patients died within 5 year. The prevalence of this dataset is $26.5 \%$. The third dataset with the name of "Diabetes" is obtained from the study of diabetes in Pima Indian population. 500 samples were identified as negative while the other 268 samples were identified as positive, which gives the prevalence of $34.9 \%$. The last dataset called "Breast" was created for breast tumor diagnosis. Within this dataset, 151 are benign samples and 47 are malignant samples, and the prevalence is $23.7 \%$.

The GWAS dataset contains 146 samples with each sample been described by more than 100,000 SNPs. Within the 146 samples 46 are labeled as geographic atrophy either central or non-central to the macula (CGA), 50 are labeled as uniocular choroidal neovascularization (Neov), and the rest 50 are the control samples. Therefore, the task is divided to classify CGA samples from the rest (which gives a prevalence of minority class of $31.5 \%$ ) and to classify Neov samples from the rest (which gives a prevalence of minority class 
of $34.2 \%$ ). In SNPs selection, we applied the selection procedure utilized by Chen et al. [32], and obtained 17 SNPs from two Linkage Disequilibrium (LD) blocks. They are rs2019727, rs10489456, rs3753396, rs380390, rs2284664, and rs1329428 from the first block, and rs4723261, rs764127, rs10486519, rs964707, rs10254116, rs10486521, rs10272438, rs10486523, 10486524 , rs10486525, and rs1420150 from the second block. Based on previous investigation of AMD [33-35], we added another six SNPs to avoid analysis bias. They are rs800292, rs1061170, rs1065489, rs1049024, rs2736911, and rs10490924. Moreover, environment factors of Smoking status and Sex are also encoded into each dataset due to their high association to the AMD development. Together, we formed the two subtype datasets with each sample represented as 25 factors.

The summary of each dataset is given in Table 1.

\section{Implementation}

We compare our particle swarm based sampling strategy with random undersampling, random oversampling, and clustering based sampling. Random undersampling and random oversampling are implemented by decreasing samples of majority class or increasing samples of minority class to match the counterpart with a uniformed possibility, respectively. Clustering based sampling is implemented as the base version of those described in [10], that is, to cluster the data samples with $k$-mean algorithm and randomly select samples of majority class according to the majority/minority ratio of each cluster and the cluster size. We used the $k$ size of 10 for $k$-mean clustering and the Euclidean distance for similarity calculation.

As per the particle swarm based hybrid system, we code the particle space as an $m$ dimension space with $m$ equals to the size of the majority samples in the training set. Different parameter settings of the particle swarm component are investigated empirically, and we fix the best combination (as shown in Table 2) for evaluation and comparison. Different classification algorithms are implemented by using APIs of the WEKA machine learning suite [28] through the main code.

Table I: Summary of the medical and biological datasets used in the experiments

\begin{tabular}{lcccc}
\hline Dataset & \# Feature & \# Negative & \# Positive & Prevalence \\
\hline Blood & 4 & 568 & 180 & $24.1 \%$ \\
Survival & 3 & 225 & 81 & $26.5 \%$ \\
Diabetes & 8 & 500 & 268 & $34.9 \%$ \\
Breast & 32 & 151 & 47 & $23.7 \%$ \\
AMD-CGA & 25 & 100 & 46 & $31.5 \%$ \\
AMD-Neov & 25 & 96 & 50 & $34.2 \%$ \\
\hline
\end{tabular}

Table 2: Parameter settings of the particle swarm based hybrid system

\begin{tabular}{lc}
\hline Parameter & Value \\
\hline Size of Classification Committee & 5 \\
Number of Evaluation Metrics & 3 \\
Size of Particle Population & 100 \\
Iteration & 150 \\
Update Rule & Sigmoid Function \\
Cognitive Constant & 1.43 \\
Social Acceleration Constant & 1.43 \\
Inertia Weight & 0.689 \\
Velocity Bound & $0.018-0.982$ \\
Fitness Weight & $w_{1}=w_{2}=w_{3}=1 / 3$ \\
\hline
\end{tabular}

\section{Results}

Tables 3, 4, 5, 6, 7, 8 provide the evaluation details of each sampling method on each dataset, respectively. All results are obtained by averaging three independent trials on each dataset. We named particle swarm based hybrid system as "PSO", random undersampling as "RU", random oversampling as "RO", and clustering based sampling as "Cluster" for convenience. For each sampling method, the evaluation results are presented with respect to 3 evaluation metrics and 10 different classification algorithms including Decision Tree (J48), 3-Nearest Neighbor (3NN), Naive Bayes (NB), Random Forest with 5 trees (RF5), Logistic Regression (LOG), 1-Nearest Neighbor (1NN), 7-Nearest Neighbor (7NN), Sequential Minimal Optimization of Support Vector Machine (SMO), Random Forest with 10 trees (RF10), and Radial Basis Function Network (RBFNet). With a careful observation it is clear that in most cases PSO achieved better classification accuracy using all three evaluation metrics in comparison with the other three sampling methods. This can be further confirmed by averaging across different classification results with respect to each evaluation metric (indicated in column "R. Avg." of Tables 3, 4, 5, 6, 7, 8). Also observed is that the improvement is essentially consistent across 10 different types of classifiers. This can be seen from the row "C. Avg." of Tables 3, 4, 5, 6, 7, 8. It should be noted that only the first five classifiers are used in PSO optimization and data sampling, while the last five classifiers are only used for evaluating the generation property of the hybrid system. Also, the evaluation is done on the independent test set through external cross validation. Therefore, it is safe to draw a conclusion that re-sampling dataset using PSO can lead to a higher data sampling quality and better generalization property. For random undersampling and random oversampling we found that random undersampling is more effective, albeit in a few cases random oversampling appear to be quite competitive. As to clustering based sampling, it performs competitively to random under- and oversampling in "Diabetes", "Breast", "AMD-CGA", and 
Table 3: Evaluation results of Blood dataset using different sampling strategies with three metrics across ten classification algorithms

\begin{tabular}{|c|c|c|c|c|c|c|c|c|c|c|c|c|}
\hline \multirow[t]{2}{*}{ Method } & \multirow[t]{2}{*}{ Metric } & \multicolumn{11}{|c|}{ Classifier } \\
\hline & & $J 48$ & 3NN & NB & RF5 & LOG & INN & 7NN & SMO & RFIO & RBFNet & R. Avg. \\
\hline \multirow[t]{4}{*}{ PSO } & AUC & 0.693 & 0.656 & 0.706 & 0.656 & 0.736 & 0.612 & 0.696 & 0.667 & 0.660 & 0.720 & 0.680 \\
\hline & FMeasure & 0.495 & 0.446 & 0.458 & 0.430 & 0.494 & 0.409 & 0.485 & 0.486 & 0.434 & 0.487 & 0.462 \\
\hline & GMean & 0.671 & 0.622 & 0.634 & 0.605 & 0.668 & 0.590 & 0.662 & 0.655 & 0.614 & 0.663 & 0.638 \\
\hline & C. Avg. & 0.620 & 0.575 & 0.599 & 0.564 & 0.633 & 0.537 & 0.614 & 0.603 & 0.569 & 0.623 & 0.593 \\
\hline \multirow[t]{4}{*}{$R U$} & AUC & 0.663 & 0.647 & 0.713 & 0.632 & 0.745 & 0.597 & 0.689 & 0.666 & 0.638 & 0.710 & 0.669 \\
\hline & FMeasure & 0.474 & 0.425 & 0.417 & 0.419 & 0.511 & 0.393 & 0.461 & 0.486 & 0.424 & 0.462 & 0.447 \\
\hline & GMean & 0.643 & 0.609 & 0.586 & 0.600 & 0.686 & 0.577 & $0.64 I$ & 0.655 & 0.605 & 0.639 & 0.624 \\
\hline & C. Avg. & 0.593 & 0.560 & 0.572 & 0.550 & 0.647 & 0.522 & 0.597 & 0.602 & 0.556 & 0.604 & 0.580 \\
\hline \multirow[t]{4}{*}{ RO } & AUC & 0.657 & 0.635 & 0.710 & 0.618 & 0.749 & 0.573 & 0.652 & 0.671 & 0.629 & 0.715 & 0.661 \\
\hline & FMeasure & 0.460 & 0.422 & 0.375 & 0.387 & 0.514 & 0.339 & 0.432 & 0.491 & 0.380 & 0.474 & 0.428 \\
\hline & GMean & 0.635 & 0.607 & 0.538 & 0.568 & 0.689 & 0.522 & 0.615 & 0.663 & $0.56 \mathrm{I}$ & 0.651 & 0.605 \\
\hline & C. Avg. & 0.584 & 0.555 & $0.54 I$ & 0.524 & 0.651 & 0.478 & 0.566 & 0.608 & 0.523 & 0.613 & 0.565 \\
\hline \multirow[t]{4}{*}{ Cluster } & AUC & 0.616 & 0.660 & 0.677 & 0.614 & $0.65 I$ & 0.571 & 0.661 & 0.658 & 0.629 & 0.711 & 0.645 \\
\hline & FMeasure & 0.449 & 0.420 & 0.382 & 0.405 & 0.429 & 0.318 & 0.414 & 0.419 & 0.348 & 0.454 & 0.404 \\
\hline & GMean & 0.587 & 0.583 & 0.556 & 0.559 & 0.560 & 0.534 & 0.616 & 0.635 & 0.608 & 0.658 & 0.590 \\
\hline & C. Avg. & 0.551 & 0.554 & 0.538 & 0.526 & 0.547 & 0.474 & 0.564 & 0.571 & 0.528 & 0.608 & 0.546 \\
\hline
\end{tabular}

Table 4: Evaluation results of Survival dataset using different sampling strategies with three metrics across ten classification algorithms

\begin{tabular}{|c|c|c|c|c|c|c|c|c|c|c|c|c|}
\hline \multirow[t]{2}{*}{ Method } & \multirow[t]{2}{*}{ Metric } & \multicolumn{11}{|c|}{ Classifier } \\
\hline & & J48 & $3 N N$ & NB & RF5 & LOG & INN & 7NN & SMO & RF I 0 & RBFNet & R. Avg. \\
\hline \multirow[t]{4}{*}{ PSO } & AUC & 0.660 & 0.626 & 0.660 & 0.668 & 0.698 & 0.614 & 0.618 & 0.617 & 0.680 & 0.700 & 0.654 \\
\hline & FMeasure & 0.495 & 0.422 & 0.495 & 0.469 & 0.542 & 0.459 & 0.421 & 0.447 & 0.464 & 0.492 & $0.47 I$ \\
\hline & GMean & $0.64 I$ & 0.580 & 0.635 & 0.618 & 0.687 & 0.612 & 0.581 & 0.587 & 0.614 & 0.643 & 0.620 \\
\hline & C. Avg. & 0.599 & 0.543 & 0.597 & 0.585 & 0.642 & 0.562 & 0.540 & 0.550 & 0.586 & 0.612 & 0.582 \\
\hline \multirow[t]{4}{*}{$\mathrm{RU}$} & AUC & 0.636 & 0.565 & 0.633 & 0.628 & 0.668 & 0.589 & 0.619 & 0.586 & 0.659 & $0.65 I$ & 0.623 \\
\hline & FMeasure & 0.482 & 0.406 & 0.459 & 0.455 & 0.486 & 0.436 & 0.429 & 0.399 & 0.460 & 0.477 & 0.449 \\
\hline & GMean & 0.626 & 0.562 & 0.598 & 0.599 & 0.637 & 0.589 & 0.587 & 0.554 & 0.608 & 0.619 & 0.598 \\
\hline & C. Avg. & 0.581 & 0.511 & 0.563 & 0.561 & 0.597 & 0.538 & 0.545 & 0.513 & 0.576 & 0.582 & 0.557 \\
\hline \multirow[t]{4}{*}{ RO } & AUC & 0.619 & 0.617 & $0.64 I$ & 0.631 & 0.684 & 0.588 & 0.602 & 0.615 & 0.639 & 0.663 & 0.631 \\
\hline & FMeasure & 0.465 & 0.433 & 0.427 & 0.389 & 0.487 & 0.354 & 0.413 & 0.411 & 0.368 & 0.459 & 0.422 \\
\hline & GMean & 0.617 & 0.579 & 0.561 & 0.553 & 0.632 & 0.514 & 0.573 & 0.547 & 0.534 & 0.608 & 0.574 \\
\hline & C. Avg. & 0.567 & 0.543 & 0.543 & 0.524 & 0.601 & 0.485 & 0.529 & 0.524 & 0.514 & 0.577 & 0.542 \\
\hline \multirow[t]{4}{*}{ Cluster } & AUC & 0.623 & 0.564 & 0.642 & 0.601 & 0.664 & 0.546 & 0.570 & 0.595 & 0.616 & 0.634 & 0.606 \\
\hline & FMeasure & 0.451 & 0.376 & 0.443 & 0.366 & 0.460 & 0.325 & 0.397 & 0.380 & 0.389 & 0.436 & 0.402 \\
\hline & GMean & 0.602 & 0.538 & 0.559 & 0.539 & 0.636 & 0.497 & 0.546 & 0.512 & 0.570 & 0.601 & 0.560 \\
\hline & C. Avg. & 0.559 & 0.493 & 0.548 & 0.502 & 0.587 & 0.456 & 0.504 & 0.496 & 0.525 & 0.557 & 0.523 \\
\hline
\end{tabular}

"AMD-Neov" datasets but relatively poor on "Blood" and "Survival" datasets.

By plotting the evaluation results with respect to different evaluation metrics (shown in Figure 4), we can see that the PSO hybrid achieved the highest accuracy within all six datasets. However, it is also clear that each evaluation metric gives a different evaluation indication. That is, a sampling method " $\mathrm{A}$ " performing worse than another method " $\mathrm{B}$ " according to certain evaluation metric may be superior to the method "B" using a different evaluation metric. By plotting the 
Table 5: Evaluation results of Diabetes dataset using different sampling strategies with three metrics across ten classification algorithms

\begin{tabular}{|c|c|c|c|c|c|c|c|c|c|c|c|c|}
\hline \multirow[t]{2}{*}{ Method } & \multirow[t]{2}{*}{ Metric } & \multicolumn{11}{|c|}{ Classifier } \\
\hline & & $J 48$ & $3 N N$ & NB & RF5 & LOG & INN & 7NN & SMO & RFIO & RBFNet & R. Avg. \\
\hline \multirow[t]{4}{*}{ PSO } & AUC & 0.746 & 0.761 & 0.808 & 0.801 & 0.827 & 0.693 & 0.793 & 0.740 & 0.817 & 0.786 & 0.777 \\
\hline & FMeasure & 0.660 & 0.618 & 0.638 & 0.662 & 0.661 & 0.612 & 0.651 & 0.662 & 0.671 & 0.639 & 0.647 \\
\hline & GMean & 0.734 & 0.698 & 0.717 & 0.736 & 0.734 & 0.691 & 0.727 & 0.738 & 0.745 & 0.719 & 0.724 \\
\hline & C. Avg. & 0.713 & 0.692 & 0.721 & 0.733 & $0.74 I$ & 0.665 & 0.724 & 0.713 & 0.744 & 0.715 & 0.716 \\
\hline \multirow[t]{4}{*}{$R U$} & AUC & 0.697 & 0.739 & 0.801 & 0.765 & 0.829 & 0.665 & 0.773 & 0.737 & 0.791 & 0.759 & 0.756 \\
\hline & FMeasure & 0.635 & 0.603 & 0.635 & 0.628 & 0.665 & 0.581 & 0.636 & 0.657 & 0.659 & 0.609 & 0.631 \\
\hline & GMean & 0.707 & 0.686 & 0.714 & 0.705 & 0.740 & 0.660 & 0.714 & 0.734 & 0.734 & 0.694 & 0.709 \\
\hline & C. Avg. & 0.680 & 0.676 & 0.717 & 0.699 & 0.745 & 0.635 & 0.708 & 0.709 & 0.728 & 0.687 & 0.699 \\
\hline \multirow[t]{4}{*}{ RO } & AUC & 0.709 & 0.722 & 0.799 & 0.774 & 0.831 & 0.653 & 0.774 & 0.735 & 0.796 & 0.797 & 0.760 \\
\hline & FMeasure & 0.634 & 0.592 & 0.628 & 0.612 & 0.665 & 0.549 & 0.621 & 0.656 & 0.616 & 0.636 & 0.622 \\
\hline & GMean & 0.713 & 0.675 & 0.705 & 0.696 & 0.739 & 0.643 & 0.699 & 0.733 & 0.696 & 0.716 & 0.702 \\
\hline & C. Avg. & 0.685 & 0.663 & 0.711 & 0.694 & 0.745 & 0.615 & 0.698 & 0.708 & 0.703 & 0.716 & 0.695 \\
\hline \multirow[t]{4}{*}{ Cluster } & AUC & 0.701 & 0.729 & 0.801 & 0.759 & 0.813 & 0.608 & 0.769 & 0.753 & 0.788 & 0.784 & 0.751 \\
\hline & FMeasure & 0.624 & 0.603 & 0.635 & 0.598 & 0.684 & 0.513 & 0.626 & 0.680 & 0.643 & 0.629 & 0.624 \\
\hline & GMean & 0.702 & 0.678 & 0.711 & 0.686 & 0.723 & 0.615 & 0.698 & 0.752 & 0.721 & 0.711 & 0.700 \\
\hline & C. Avg. & 0.676 & 0.670 & 0.716 & 0.681 & 0.740 & 0.579 & 0.698 & 0.728 & 0.717 & 0.708 & 0.692 \\
\hline
\end{tabular}

Table 6: Evaluation results of Breast dataset using different sampling strategies with three metrics across ten classification algorithms

\begin{tabular}{|c|c|c|c|c|c|c|c|c|c|c|c|c|}
\hline \multirow[t]{2}{*}{ Method } & \multirow[t]{2}{*}{ Metric } & \multicolumn{11}{|c|}{ Classifier } \\
\hline & & $J 48$ & $3 N N$ & NB & RF5 & LOG & INN & 7NN & SMO & RFIO & RBFNet & R. Avg. \\
\hline \multirow[t]{4}{*}{ PSO } & AUC & 0.580 & 0.610 & 0.602 & 0.603 & 0.736 & 0.570 & 0.625 & 0.670 & 0.637 & 0.549 & 0.618 \\
\hline & FMeasure & 0.418 & 0.423 & 0.393 & 0.369 & 0.489 & 0.394 & 0.425 & 0.487 & 0.392 & 0.359 & 0.415 \\
\hline & GMean & 0.593 & 0.599 & 0.577 & 0.550 & 0.661 & 0.553 & 0.595 & 0.660 & 0.577 & 0.544 & 0.591 \\
\hline & C. Avg. & 0.530 & 0.544 & 0.524 & 0.507 & 0.629 & 0.506 & 0.548 & 0.606 & 0.535 & 0.484 & $0.54 I$ \\
\hline \multirow[t]{4}{*}{ RU } & AUC & 0.562 & 0.597 & 0.587 & 0.604 & 0.722 & 0.515 & 0.612 & 0.650 & 0.639 & 0.568 & 0.606 \\
\hline & FMeasure & 0.393 & 0.407 & 0.378 & 0.403 & 0.479 & 0.345 & 0.422 & 0.466 & 0.399 & 0.388 & 0.408 \\
\hline & GMean & 0.552 & 0.583 & 0.558 & 0.571 & 0.656 & 0.502 & 0.597 & 0.637 & 0.581 & 0.568 & 0.581 \\
\hline & C. Avg. & 0.502 & 0.529 & 0.508 & 0.526 & 0.619 & 0.454 & 0.544 & 0.584 & 0.540 & 0.508 & 0.532 \\
\hline \multirow[t]{4}{*}{ RO } & AUC & 0.569 & 0.564 & 0.596 & 0.593 & 0.789 & 0.544 & 0.582 & 0.688 & 0.639 & 0.480 & 0.604 \\
\hline & FMeasure & 0.327 & 0.391 & 0.384 & 0.354 & 0.560 & 0.325 & 0.382 & 0.509 & 0.297 & 0.286 & 0.382 \\
\hline & GMean & 0.508 & 0.565 & 0.569 & 0.522 & 0.701 & 0.512 & 0.560 & 0.683 & 0.452 & 0.475 & 0.555 \\
\hline & C. Avg. & 0.468 & 0.507 & 0.516 & 0.490 & 0.683 & 0.460 & 0.508 & 0.627 & 0.463 & 0.414 & 0.514 \\
\hline \multirow[t]{4}{*}{ Cluster } & AUC & 0.543 & 0.616 & 0.538 & 0.554 & 0.711 & 0.547 & 0.603 & $0.64 I$ & 0.586 & 0.528 & 0.587 \\
\hline & FMeasure & 0.384 & 0.402 & 0.356 & 0.325 & 0.466 & $0.37 \mid$ & 0.417 & 0.450 & 0.342 & 0.332 & 0.385 \\
\hline & GMean & 0.579 & 0.585 & 0.550 & 0.508 & 0.648 & 0.527 & 0.583 & 0.638 & 0.540 & 0.529 & 0.569 \\
\hline & C. Avg. & 0.502 & 0.534 & 0.481 & 0.462 & 0.608 & 0.482 & 0.534 & 0.576 & 0.489 & 0.463 & 0.514 \\
\hline
\end{tabular}


Table 7: Evaluation results of AMD-CGA dataset using different sampling strategies with three metrics across ten classification algorithms

\begin{tabular}{|c|c|c|c|c|c|c|c|c|c|c|c|c|}
\hline \multirow[t]{2}{*}{ Method } & \multirow[t]{2}{*}{ Metric } & \multicolumn{11}{|c|}{ Classifier } \\
\hline & & $J 48$ & $3 N N$ & NB & RF5 & LOG & INN & 7NN & SMO & RFIO & RBFNet & R. Avg. \\
\hline \multirow[t]{4}{*}{ PSO } & AUC & 0.609 & 0.559 & 0.606 & 0.591 & 0.566 & 0.599 & 0.590 & 0.572 & 0.573 & 0.540 & 0.581 \\
\hline & FMeasure & $0.48 I$ & 0.462 & 0.489 & 0.464 & 0.448 & 0.485 & 0.468 & 0.460 & 0.465 & 0.455 & 0.468 \\
\hline & GMean & 0.580 & 0.557 & 0.572 & 0.551 & 0.538 & 0.576 & 0.573 & 0.550 & 0.545 & 0.539 & 0.558 \\
\hline & C. Avg. & 0.557 & 0.526 & 0.556 & 0.535 & 0.517 & 0.553 & 0.544 & 0.527 & 0.528 & 0.511 & 0.536 \\
\hline \multirow[t]{4}{*}{$\mathrm{RU}$} & AUC & 0.569 & 0.547 & 0.549 & 0.594 & 0.567 & 0.569 & 0.579 & 0.556 & 0.604 & 0.570 & 0.570 \\
\hline & FMeasure & 0.434 & 0.457 & 0.439 & 0.476 & 0.453 & 0.439 & 0.446 & 0.417 & 0.456 & 0.446 & 0.446 \\
\hline & GMean & 0.538 & 0.556 & 0.538 & 0.580 & 0.551 & 0.547 & 0.553 & 0.523 & 0.550 & 0.549 & 0.549 \\
\hline & C. Avg. & 0.514 & 0.520 & 0.509 & 0.550 & 0.524 & 0.518 & 0.526 & 0.499 & 0.537 & 0.522 & 0.522 \\
\hline \multirow[t]{4}{*}{ RO } & AUC & 0.566 & 0.568 & 0.565 & 0.597 & 0.581 & 0.569 & 0.586 & 0.558 & 0.576 & 0.586 & 0.575 \\
\hline & FMeasure & 0.394 & 0.405 & 0.375 & 0.442 & 0.420 & 0.415 & 0.407 & 0.358 & 0.421 & 0.428 & 0.407 \\
\hline & GMean & 0.523 & 0.530 & 0.505 & 0.564 & 0.544 & 0.539 & 0.537 & 0.490 & 0.547 & 0.555 & 0.533 \\
\hline & C. Avg. & 0.494 & 0.501 & 0.482 & 0.534 & 0.515 & 0.508 & 0.510 & 0.469 & 0.515 & 0.523 & 0.505 \\
\hline \multirow[t]{4}{*}{ Cluster } & AUC & 0.519 & 0.595 & 0.560 & 0.601 & 0.580 & 0.580 & 0.566 & 0.545 & 0.581 & 0.576 & 0.570 \\
\hline & FMeasure & 0.306 & 0.343 & 0.358 & 0.357 & 0.332 & 0.270 & 0.301 & 0.368 & 0.387 & 0.339 & 0.445 \\
\hline & GMean & 0.499 & 0.558 & 0.532 & 0.566 & 0.548 & 0.569 & 0.546 & 0.511 & 0.554 & 0.548 & 0.543 \\
\hline & C. Avg. & $0.44 I$ & 0.499 & 0.483 & 0.508 & 0.487 & 0.473 & 0.47 I & 0.475 & 0.507 & 0.488 & 0.519 \\
\hline
\end{tabular}

Table 8: Evaluation results of AMD-Neov dataset using different sampling strategies with three metrics across ten classification algorithms

\begin{tabular}{|c|c|c|c|c|c|c|c|c|c|c|c|c|}
\hline \multirow[t]{2}{*}{ Method } & \multirow[t]{2}{*}{ Metric } & \multicolumn{11}{|c|}{ Classifier } \\
\hline & & $J 48$ & $3 \mathbf{N N}$ & NB & RF5 & LOG & INN & 7NN & SMO & RFIO & RBFNet & R. Avg. \\
\hline \multirow[t]{4}{*}{ PSO } & AUC & $0.68 I$ & 0.659 & 0.661 & 0.662 & 0.678 & 0.656 & 0.694 & 0.628 & 0.686 & 0.672 & 0.668 \\
\hline & FMeasure & 0.549 & 0.557 & 0.537 & 0.566 & 0.545 & 0.556 & 0.572 & 0.559 & 0.552 & 0.559 & 0.555 \\
\hline & GMean & 0.622 & 0.628 & 0.619 & 0.643 & 0.626 & 0.630 & 0.648 & 0.637 & 0.631 & 0.631 & 0.632 \\
\hline & C. Avg. & 0.617 & 0.615 & 0.605 & 0.624 & 0.616 & 0.614 & 0.638 & 0.608 & 0.623 & 0.621 & 0.618 \\
\hline \multirow[t]{4}{*}{$R U$} & AUC & 0.652 & 0.627 & 0.625 & 0.622 & 0.635 & 0.649 & 0.622 & 0.619 & 0.663 & 0.631 & 0.635 \\
\hline & FMeasure & 0.549 & 0.526 & 0.524 & 0.534 & 0.519 & 0.531 & 0.543 & 0.529 & 0.561 & 0.539 & 0.536 \\
\hline & GMean & 0.637 & 0.602 & 0.601 & 0.609 & 0.596 & 0.615 & 0.615 & 0.604 & 0.636 & 0.612 & 0.613 \\
\hline & C. Avg. & 0.613 & 0.585 & 0.583 & 0.588 & 0.583 & 0.598 & 0.593 & 0.584 & 0.620 & 0.594 & 0.595 \\
\hline \multirow[t]{4}{*}{ RO } & AUC & 0.643 & 0.643 & 0.646 & 0.659 & 0.635 & 0.655 & 0.638 & 0.632 & 0.660 & 0.657 & 0.647 \\
\hline & FMeasure & 0.507 & 0.542 & 0.491 & 0.516 & 0.498 & 0.516 & 0.521 & 0.506 & 0.534 & 0.531 & 0.516 \\
\hline & GMean & 0.602 & 0.629 & 0.589 & 0.610 & 0.599 & 0.612 & 0.612 & 0.598 & 0.624 & 0.623 & 0.610 \\
\hline & C. Avg. & 0.584 & 0.605 & 0.575 & 0.595 & 0.577 & 0.594 & 0.590 & 0.579 & 0.606 & 0.603 & 0.591 \\
\hline \multirow[t]{4}{*}{ Cluster } & AUC & 0.656 & 0.624 & 0.627 & 0.629 & 0.625 & 0.652 & 0.644 & 0.594 & 0.642 & 0.638 & 0.633 \\
\hline & FMeasure & 0.551 & 0.524 & 0.502 & 0.538 & 0.506 & 0.521 & 0.546 & 0.504 & 0.536 & 0.537 & 0.527 \\
\hline & GMean & $0.64 I$ & 0.605 & 0.587 & 0.624 & 0.591 & 0.610 & 0.630 & 0.585 & 0.620 & 0.621 & 0.611 \\
\hline & C. Avg. & 0.616 & 0.584 & 0.572 & 0.597 & 0.574 & 0.594 & 0.607 & $0.56 I$ & 0.599 & 0.599 & 0.590 \\
\hline
\end{tabular}



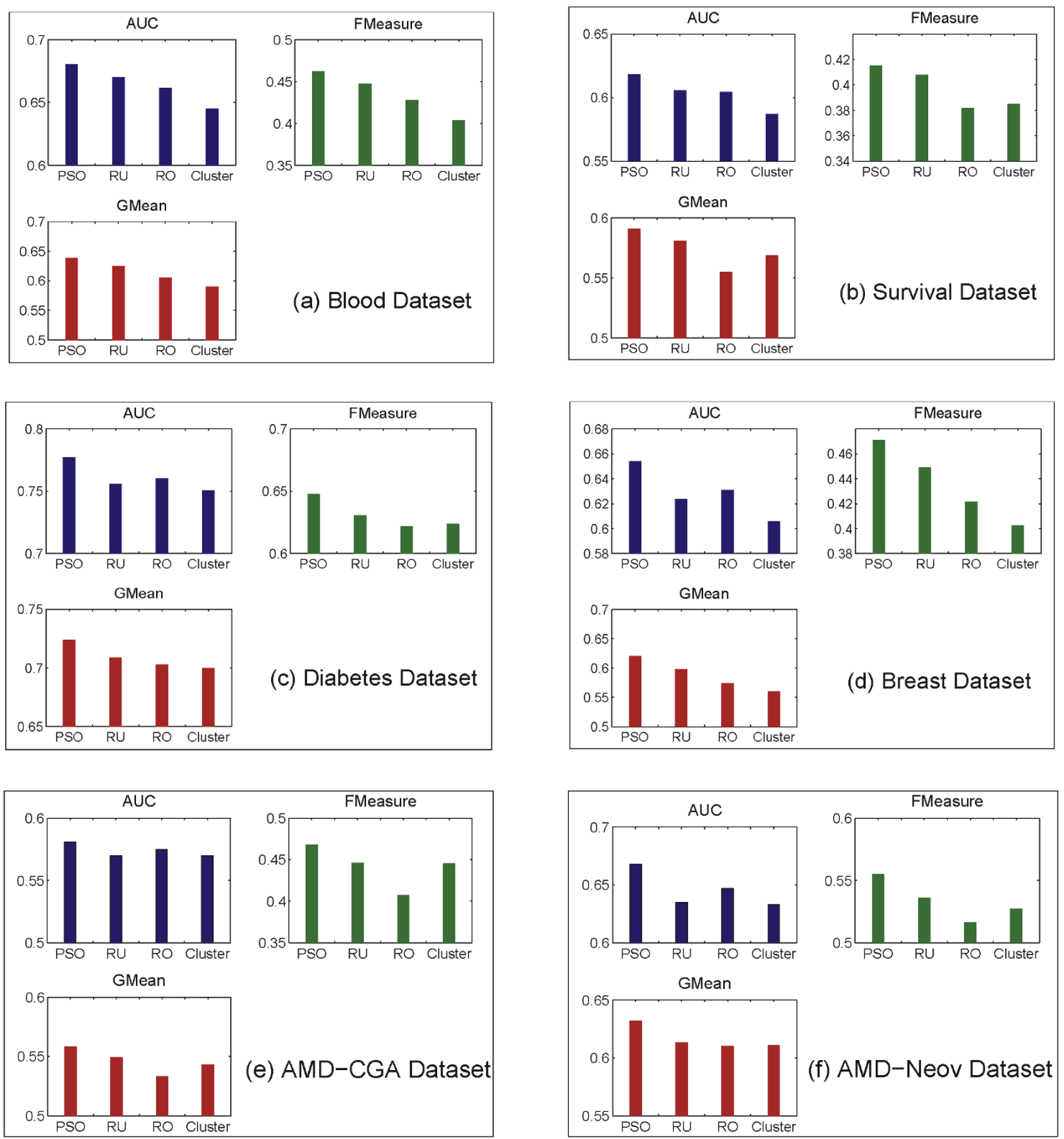

Figure 4

Figure 4

Comparison of each sampling method with respect to different evaluation metrics.

evaluation results with respect to different classification algorithms (in Figure 5), it is readily noticed that different classifiers also perform differently among these datasets. But within a given dataset, there seems to have certain data-classifier correlation regardless which type of the sampling method is used. Interestingly, logistic classifier seems to be quite effective, while $1 \mathrm{NN}$ appears to be the most unsuccessful one. 
(a) Blood Dataset

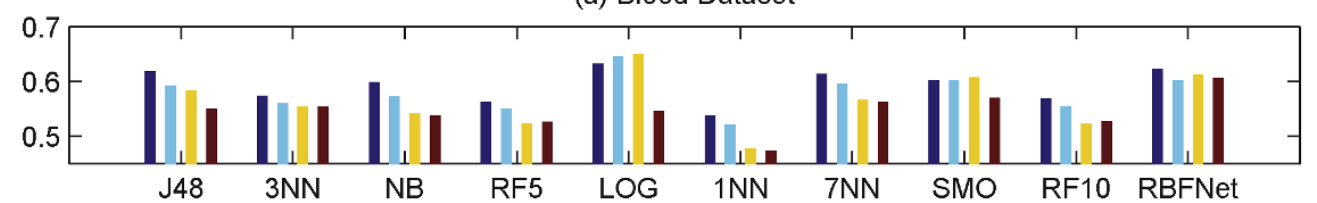

(b) Survival Dataset
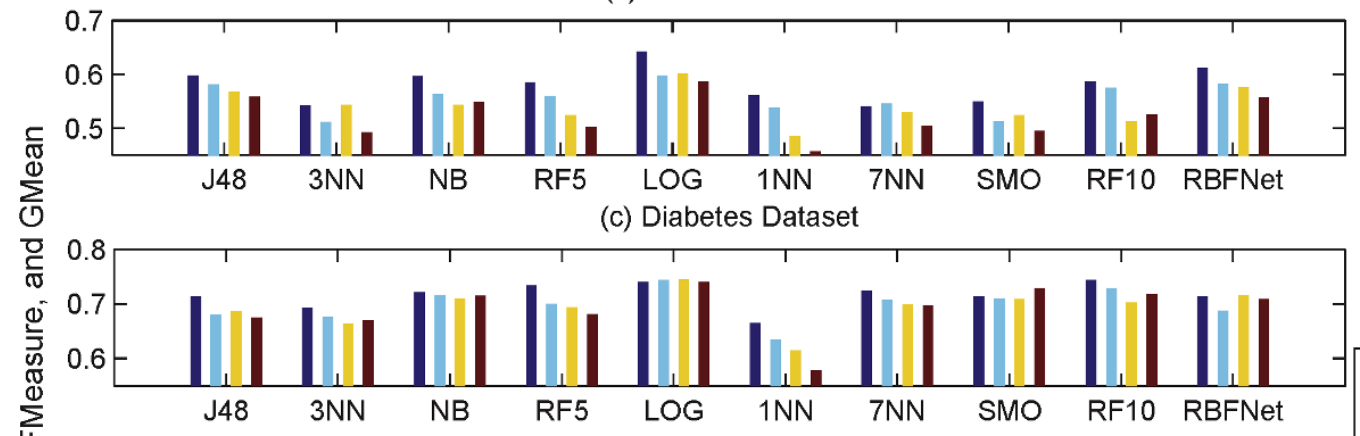

(d) Breast Dataset

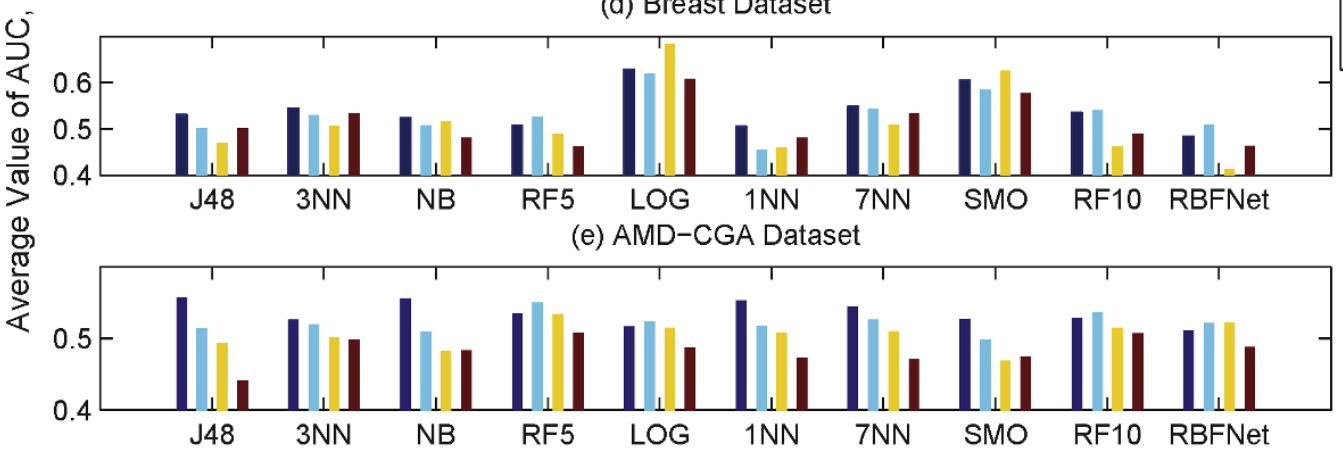

(f) AMD-Neov Dataset

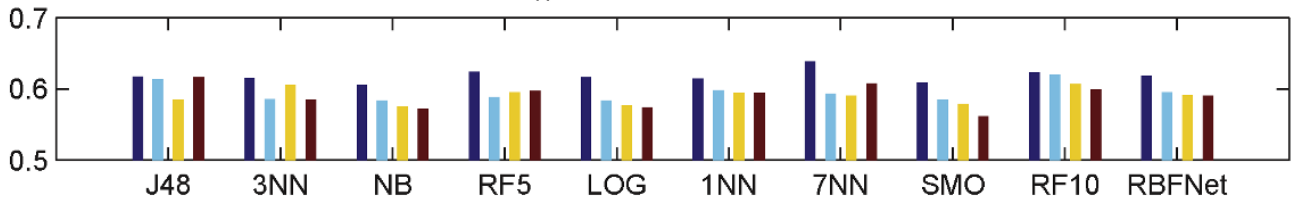

Figure 5

Figure 5

Comparison of each sampling method with respect to different classification algorithms.

With above observation, it is clear that the evaluation of different data sampling strategies is compounded by different classification algorithms and evaluation metrics. Therefore, relying on a sole classifier or evaluation measure for imbalanced data sampling could potentially lead to the loss of generalization property. Caution should be drawn when a claim is made on the basis of a single type of classifier or evaluation metric.

\section{Conclusion}

In this work, several popular sampling methods are investigated on imbalanced medical and biological data classification. A particle swarm based hybrid method is proposed to improve the overall classification accuracy. The experimental results on four medical datasets and a GWAS dataset illustrated the effectiveness of the proposed method. This is quantified in our experiments by using three evaluation metrics across 10 different classification algorithms.

The study demonstrates that with a proper modification feature selection algorithms can be tailored for imbalanced data sampling. In addition to being self-adaptable to different datasets, the proposed hybrid system is quite flexible, allowing different classifiers and evaluation 
components to be easily integrated for any specific problem at hand. The imbalanced data sampling problem is ubiquitous in clinical and medical diagnoses as well as gene function predication and protein classification [36,37]. The proposed hybrid system can not only recover the power of classifiers on imbalance data classification but also indicate the relative importance of samples from majority class in contrast to samples from minority class. This information could be used for further biological and medical investigations which may result in the discovery of new conditions or disease subtypes. We anticipate that such a hybrid formulation can provide a new means for tackling imbalanced data problems introduced in these applications.

\section{Availability}

The PSO sampling system is implemented in Java and is available from: http://www.cs.usyd.edu.au/ yangpy/ software/Sampling

\section{Competing interests}

The authors declare that they have no competing interests.

\section{Authors' contributions}

PY conceived the study and drafted the manuscript. PY and LX designed and implemented the algorithms, performed the experiments. BBZ, ZZ, and AYZ revised the manuscript critically and introduced the problem initially.

\section{Note}

Other papers from the meeting have been published as part of BMC Bioinformatics Volume 10 Supplement 15, 2009: Eighth International Conference on Bioinformatics (InCoB2009): Bioinformatics, available online at http://www.biomedcentral.com/1471-2105/10? issue $=\mathrm{S} 15$.

\section{Acknowledgements}

The authors would like to thank Joshua WK Ho from NICTA and SIT, The University of Sydney for inspiring discussions and valuable suggestions. PY is supported by a NICTA International Postgraduate Award (NIPA) and a NICTA Research Project Award (NRPA).

This article has been published as part of BMC Genomics Volume 10 Supplement 3, 2009: Eighth International Conference on Bioinformatics (InCoB2009): Computational Biology. The full contents of the supplement are available online at http://www.biomedcentral.com//47/-2/64//0? issue $=\mathrm{S} 3$.

\section{References}

I. Velez D, White B, Motsinger A, W B, Ritchie M, Williams S and Moore J: A Balanced Accuracy Function for Epistasis Modeling in Imbalanced Datasets using Multifactor Dimensionality Reduction. Genetic Epidemiology 2007, 31:306-3 I5.
2. Cohen G, Hilario M, Sax H, Hugonnet $S$ and Geissbuhler $A$ : Learning from Imbalanced Data in Surveillance of Nosocomial Infection. Artificial Intelligence in Medicine 2006, 37:7-18.

3. Yang F, Wang $\mathrm{H}, \mathrm{Mi} \mathrm{H}$, Lin $\mathrm{C}$ and Cai W: Using Random Forest for Reliable Classification and Cost-Sensitive Learning for Medical Diagnosis. BMC Bioinformatics 2009, 10:S22.

4. Mazurowski M, Habas P, Zurada J, Lo J, Baker J and Tourassi D: Training Neural Network Classifiers for Medical Decision Making: The Effects of Imbalanced Datasets on Classification Performance. Neural Networks 2008, 21 (2-3):427-436.

5. Kapp A, Jeffrey S, Langerød A, Børresen-Dale A, Han W, Hoh D, Bukholm I, Nicolau M, Brown P and Tibshirani R: Discovery and validation of breast cancer subtypes. BMC Genomics 2006, 7:23I.

6. Chawla N, Japkowicz N and Kotcz A: Editorial: Special Issue on Learning from Imbalanced Data Sets. SIGKDD Explorations 2004, 6: I-6.

7. Weiss G: Mining with Rarity: A Unifying Framework. SIGKDD Explorations 2004, 6:7-19.

8. Han H, Wang W and Mao B: Borderline-SMOTE: A New Oversampling Method in Imbalanced Data Sets Learning. Proceedings of Advances in Intelligent Computing 2005, 878-887.

9. Khoshgoftaar T, Seiffert $C$ and Van Hulse J: Hybrid Sampling for Imbalanced Data. Proceedings of IRl'08 2008, 202-207.

10. Yen $\mathrm{S}$ and Lee Y: Cluster-based Under-sampling Approaches for Imbalanced Data Distributions. Expert Systems with Applications 2009, 36(3):57I8-5727.

II. Japkowicz N and Stephen S: The Class Imbalance Problem: A Systematic Study. Intelligent Data Analysis 2002, 6(5):429-449.

12. Barandela R, Sanchez J, Garcia $V$ and Rangel E: Strategies for Learning in Class Imbalance Problems. Pattern Recognition 2003, 36:849-851.

13. Chawla N, Bowyer K, Hall L and Kegelmeyer W: SMOTE: Synthetic Minority Over-sampling Technique. Journal of Artificial Intelligence Research 2002, |6:32|-357.

14. Zhang J and Mani I: kNN Approach to Unbalanced Data Distributions: A Case Study Involving Informaiton Extraction. Proceedings of the ICML'O3 Workshop on Learning from Imbalanced Datasets 2003.

15. Van Hulse J, Khoshgoftaar $T$ and Hapolitano A: Experimental Perspectives on Learning from Imbalanced Data. Proceedings of the 24th ICML 2007, 935-942.

16. Zhang $Z$ and Yang P: An ensemble of classifiers with genetic algorithm based feature selection. IEEE Intelligent Informatics Bulletin 2008, 9: 18-24.

17. Kennedy J and Eberhart R: Particle swarm optimization. Proceedings of IEEE International Conference on Neural Networks 1995, 1942-1948.

18. Kennedy J and Eberhart R: A Discrete Binary Version of the Particle Swarm Algorithm. Proceedings of IEEE International Conference on Systems Man and Cybernetics 1997, 4104-4108.

19. Batista G, Prati R and Monard M: A Study of the Behavior of Several Methods for Balancing machine Learning Training Data. SIGKDD Explorations 2004, 6:20-24.

20. Drummond $C$ and Holte R: C4.5, class imbalance, and cost sensitiveity: Why under-sampling beats over-sampling. Proceedings of the ICML'03 Workshop on Learning from Imbalanced Datasets 2003.

21. Barandela R and Valdovinos R: The Imbalanced Training Sample Problem: Under or over Sampling? Proceedings of SSPR\&SPR 2004, LNCS 3/38 2004, 806-8I4.

22. Lee Z: An Integrated Algorithm for Gene Selection and Classification Applied to Microarray Data of Ovarian Cancer. Artificial Intelligence in Medicine 2008, 42:8I-93.

23. Xu R, Wunsch D II and Frank R: Inference of Genetic Regulatory Networks with Recurrent Neural Network Models Using Particle Swarm Optimiztion. IEEE/ACM Transactions on Computational Biology and Bioinformatics 2007, 4(4):68I-692.

24. Rasmussen T and Krink T: Improved Hidden Markov Model Training for Multiple Sequence Alignment by a Particle Swarm Optimization-Evolutionary Algorithm Hybrid. Biosystems 2003, 72(I-2):5-17.

25. Lee S, Soak S, Oh S, Pedrycz W and Jeon M: Modified Binary Particle Swarm Optimization. Progress in Natural Science 2008, 18:1161-1166.

26. Cantor $S$ and Kattan M: Determining the Area Under the ROC Curve for a Binary Diagnostic Test. Medical Decision Making 2000, 20:468-470. 
27. Bradley A: The use of the Area Under the ROC Curve in the Evaluation of Machine Learning Algorithms. Pattern Recognition 1997, 30: II45-1159.

28. Witten IH and Frank MD: Data Mining: Practical Machine Learning Tools and Techniques (Second Edition) Elsevier; 2005.

29. Breiman L: Random Forests. Machine Learning 200I, 45:5-32.

30. UCI Repository of machine learning databases. http://archive. ics.uci.edu/ml/.

31. Klein RJ, Zeiss C, Chew EY, Tsai JY, Sackler HCRS, Henning AK SanGiovanni JP, Mane SM, Mayne ST, Bracken MB, Ferris FL, Ott J, Barnstable $\mathrm{C}$ and Hoh J: Complement factor $\mathbf{H}$ polymorphism in age-related macular degeneration. Science 2005, 308:385-389.

32. Cheng $X$, Zhang $M$ and Zhang $H$ : A forest-based approach to identifying gene and gene-gene interactions. Proc Natl Acad Sci U S A 2007, 104(49): 19|99-19203.

33. Haines JL, Hauser MA, Schmidt S, Scott WK and Olson LM: Complement factor $H$ variant increases the risk of agerelated macular degeneration. Science 2005, 308:4 I9-42I.

34. Schmidt S, Hauser MA, Scott WK, Postel EA and Agarwal A: Cigarette smoking strongly modifies the association of LOC 387715 and age-related macular degeneration. Am J Hum Genet 2006, 78:852-864.

35. Fisher S, Rivera A, Fritsche L, Babadjanova G, Petrov S and Weber B: Assessment of the contribution of CFH and chromosome 10q26 AMD susceptibility loci in a Russian population isolate. British Journal of Ophthalmology 2007, 91:576-578.

36. Zhao X, Li X, Chen L and Aihara K: Protein Classification with Imbalanced Data. Proteins 2008, 70(4): I I25-I I 32.

37. Zhao $X$, Wang $Y$, Chen $L$ and Aihara $K$ : Gene Function Prediction Using Labeled and Unlabeled Data. BMC Bioinformatics 2008, 9:57.

Publish with Bio Med Central and every scientist can read your work free of charge

"BioMed Central will be the most significant development for disseminating the results of biomedical research in our lifetime. "

Sir Paul Nurse, Cancer Research UK

Your research papers will be:

- available free of charge to the entire biomedical community

- peer reviewed and published immediately upon acceptance

- cited in PubMed and archived on PubMed Central

- yours - you keep the copyright
BioMedcentral 